\title{
Chronic Lymphocytic Inflammation with Pontine Perivascular Enhancement Responsive to
}

Steroids (CLIPPERS) in children - a highly aggressive condition

Mario Sa1', Lydia Green², Omar Abdel-Mannan¹, WK 'Kling' Chong³ ${ }^{3}$ Thomas Jacques', Antonia Clarke ${ }^{5}$, AnneMarie Childs², Ming Lim', ${ }^{6,7}$ Cheryl Hemingway', Yael Hacohen ${ }^{1,8}$

1. Paediatric Neurology, Great Ormond Street Hospital for Children, London, UK

2. Department of Paediatric Neurology, Leeds Teaching Hospitals NHS Trust, Leeds, UK

3. Paediatric Neuroradiology, Great Ormond street Hospital or Children, London, UK.

4. Department of Histopathology, Great Ormond Street Hospital for Children, London, UK.

5. Department of Paediatric Neurology, St Georges Healthcare NHS Trust, London, UK

6. Children's Neurosciences, Evelina London Children's Hospital @ Guy's and St Thomas' NHS Foundation Trust, King's Health Partners Academic Health Science Centre, London, UK

7. Faculty of Life Sciences and Medicine, King's College London, UK

8. Department of Neuroinflammation, Queen Square MS Centre, UCL Institute of Neurology, London, UK

${ }^{*}$ Corresponding author

Address

Dr Yael Hacohen, Department of Neuroinflammation, Queen Square MS Centre, UCL Institute of Neurology, London, UK

Email: y.hacohen@ucl.ac.uk

Running head: CLIPPERS - Report of three paediatric cases

Word Count: 1485

Abstract: 196

Title characters: 131

References: 20

Table: 1

Figure: 1 


\section{Abstract}

Objective: To describe three children with Chronic Lymphocytic Inflammation with Pontine Perivascular Enhancement Responsive to Steroids (CLIPPERS).

Background: CLIPPERS is an inflammatory condition characterised by pontine-predominant encephalomyelitis with nodular contrast enhancement in the rhombencephalic structures and a relapsing-remitting course responsive to steroids.

Cases report: Three patients (age 3yr, 13yr and 14yr) presented with ataxia, dysarthria and multiple cranial neuropathies. All patients demonstrated bilateral nodular lesions with contrast enhancement in the brainstem and cerebellum on MRI and perivascular lymphocytes and macrophages infiltrates on brain biopsies. Despite a good response to corticosteroid treatment initially, patients became steroid dependent with frequent relapses and were started on different immune-modulating treatments.

Natalizumab and IV immunoglobulin stopped patient's 1 neurological disease progression but he died aged 17 years from respiratory complications. Patient 2 continued to have disease relapses and was diagnosed with EBV driven B-cell lymphoma 3 years following symptom onset. Patient 3 failed to respond to treatment and died 4 years post diagnosis.

Conclusion: By contrast to adult reports, children seem to have an aggressive disease with poor response to immunotherapy over time. Earlier use of newer immunotherapeutic agents such as Natalizumab may be beneficial. Nevertheless, the potential side effects need to be considered carefully.

\section{Key words:}

CLIPPERS, paediatric, perivascular inflammation, natalizumab 


\section{Introduction}

A series of cases with corticosteroid responsive perivascular cerebellar and brainstem inflammation were first described in 20101, and termed chronic lymphocytic inflammation with pontine perivascular enhancement responsive to steroids (CLIPPERS). Other cases have been reported worldwide since. Typically, this pontine-predominant encephalomyelitis shows a pattern of nodular contrast enhancement or 'peppering' in the rhombencephalic structures, particularly brachium pontis, pons and cerebellum on MRI, and a predominantly $\mathrm{T}$ lymphocytic, perivascular infiltrate with parenchymal extension on CNS biopsy. In the adult population, the disease has a clinical and radiological relapsing remitting course, responsive to steroids or other immunomodulation treatments. ${ }^{2,3}$

Until recently, only a few cases of paediatric CLIPPERS have been reported ${ }^{4-7}$ and only one case confirmed with brain biopsy? ${ }^{7}$. Disease progression and outcome was thought to be similar to that in adult patients with no progressive course observed and sequelae correlated with severe relapses. ${ }^{2}$

We report three children with clinical, radiological and pathological phenotype consistent with CLIPPERS. In contrast to the favourable outcome reported in the adult literature, all three patients had a severe disease progression with frequent relapses and two patients died.

Written informed consent for the publication of the case descriptions was obtained for all three patients.

\section{Case 1:}

A previously well 3-year-old boy with a normal antenatal and developmental profile, presented with subacute onset truncal ataxia, slurred speech, and drooling. He had an unsteady broad-based gait, bilateral VI nerve palsies, reduced tone with hard to elicit reflexes and resting tremor with past pointing. Brain MRI demonstrated bilateral disease of the dorsal pons, thalamus, cerebellar white matter, with a punctate pattern of contrast enhancement (Figure $1 \mathrm{~A}$ and $\mathrm{B}$ ). CSF was acellular with no oligoclonal bands and normal lactate. Lesional brain biopsy revealed prominent perivascular lymphocytes and macrophages infiltrates suggestive of CLIPPERS (Figure 1 I). He was treated with intravenous methylprednisolone (IVMP) with dramatic improvement of symptoms. Over the next few years, he had multiple clinical relapses with similar symptomatology. He required repeated boluses of IVMP and oral prednisolone tapers, and each time the steroid course was almost complete he developed further relapses. At 6 years of age, azathioprine was started as a steroid sparing agent and steroids were completely weaned off briefly for the first time two years later. However, further relapses starting at age 9 years required re-starting of steroids. At age 12 years he developed osteoporosis and a vertebral fracture leading to cessation of steroid therapy. At age 16 years he was started on eight weekly 
natalizumab infusions and intravenous immunoglobulin (IVIG), which stopped the neurological disease progression. However, the patient died at age 17 from treatment related infection.

\section{Case 2:}

A previously well 13-year-old boy with a normal antenatal and developmental profile, presented with a six-week progressive history of ataxia and dysarthria. Brain MRI showed bilateral nodular lesions with contrast enhancement in the midbrain, brainstem, basal ganglia, and posterior limb of the internal capsule (Figure 1, E and F). CSF analysis showed persistent slightly elevated proteins, no cells or oligoclonal bands and normal lactate. He was treated with five days IVMP followed by oral prednisolone with a good response. He then developed significant steroid related side effects and these were tapered and stopped. After cessation of treatment he represented with ataxia, dysarthria in addition to drowsiness, challenging behaviour and bladder dysfunction. On examination, he showed increased tone in his lower limbs with clonus, brisk reflexes and upgoing plantar reflex on the right side. Over the next months he continued to be treated with IVMP cycles and oral prednisolone with only transient improvement and obvious steroids side effects. Eight months after presentation mycophenolate mofetil was started as a steroid sparing agent. However, as he continued to be symptomatic he also had IVIG and plasma exchange (PLEX) and later required infliximab infusions every eight weeks. At this point a brain biopsy showed an EBV driven B-cell lymphoma. (Figure 1, J)

\section{Case 3:}

A previously well 14-year-old girl presented with acute encephalopathy and multiple cranial neuropathies after a viral prodrome. She had been fit and well prior to this episode although slight cognitive impairment had been noticed. Brain MRI showed diffuse cortical grey and white matter, T2 hyperintense signal in the left middle cerebellar peduncle and dorsal pons (Figure 1, G and $H$ ). She was treated with IVMP, IVIG, and then oral prednisolone was continued. A month later she had urinary symptoms and a repeat MRI scan showed worsening of lesions, but no spinal cord lesions were found. She received IVMP again followed by oral prednisolone. While on a reducing dose regimen (3 months later) she relapsed clinically with facial weakness and marked truncal ataxia. MRI scan showed caudal root enhancement and new lesions in cerebellum. Brain biopsy showed non-specific inflammation markers. She was started on azathioprine along with IVMP and IVIG and 2 months later she relapsed with new lesions in the midbrain. She had multiple relapses and she failed to respond to 12 cycles of cyclophosphamide, PLEX and died at 47 months after initial diagnosis. 
All patients had infective, inflammatory and metabolic screens with negative results. These included plasma and urine metabolic workup, viral titres, CSF (negative for cells, viral PCR, oligoclonal bands, MOG and AQP4 antibodies), genetic testing for white matter disorders (cases 1 and 2) and muscle biopsy (patient 1).

\section{Discussion:}

We describe three biopsy proven paediatric cases of CLIPPERS. All patients fulfilled the published diagnostic criteria of CLIPPERS ${ }^{8}$. In these three cases the disease progression was more severe than expected considering previously reported cases, both from adults $1,3,4,9,10$ and paediatric ${ }^{4-7}$ patients (Table 1). Two of the patients described died after several disease relapses and the third one developed CNS B-cell lymphoma.

The imaging features with the speckled appearance of the pontine lesions together with the striking steroid responsiveness supported the diagnosis of CLIPPERS on all three cases. This diagnosis was confirmed with the findings of chronic perivascular inflammatory cell infiltrate in the brain biopsies.

As previously reported ${ }^{9,11}$ both MRI and biopsy findings suggest that cerebral legions in CLIPPERS are more diffusely spread than just the brainstem, with significant inflammatory activity likely in other areas of the brain and the spinal cord. Other possible diagnoses may resemble the radiological appearance of CLIPPERS, warranting the need for biopsy ${ }^{12}$. In view of the rarity of this syndrome in the paediatric population with only three previously reported cases, biopsy was used to exclude other alternative diagnosis such as isolated CNS vasculitis, primary CNS lymphoma, neurosarcoidosis and slow viruses.

All patients experienced recurrence of both symptoms and radiological progression quickly after taper of steroid. This was consistent with previous reports both in adults ${ }^{1}$ and paediatric patients 5,7 . Brain biopsies revealed neuro-axonal injury in addition to extensive lympho-histiocytic involvement, which may explain the disability and cerebral atrophy. The progressive loss of tissue integrity over time, likely to result in secondary neuro-axonal injury, may explain the reduced response to immunotherapies over time. This may not be disease specific as It has been previously shown in patients with other inflammatory conditions that an early disease onset may lead to more severe acute axonal injury, brain atrophy and a worse clinical outcome. ${ }^{13}$

After several clinical and imagological relapses and important side effects from prolonged steroid treatment, patient 1 was started on eight weekly natalizumab infusions, a humanized monoclonal antibody against cell adhesion molecule a4-integrin. This treatment appeared to halt neurological 
disease progression. Natalizumab has been extensively used as a disease-modifying therapy of adults and children with multiple sclerosis. ${ }^{14}$ Only three other CLIPPERS cases were reported to be treated with monoclonal antibodies, all with rituximab an anti-CD20 monoclonal antibody $4,7,10$, all with positive outcomes.

The association between CLIPPERS and primary central nervous system B-cell lymphoma has been previously described in adult patients. ${ }^{15,16}$ The exact connection between these two conditions is not completely understood and different explanations have been hypothesised. The first is that CLIPPERS is a pre-malignant state representing an host immune response preventing the clonal proliferation of malignant cells. ${ }^{17}$ The second hypothesis is that B-cell lymphoma is secondary to the immunosuppressive treatment. Immunomodulatory therapy-induced lymphoproliferative disorders represent a heterogeneous group of lymphoid cell disorders that occur secondary to iatrogenic immune dysfunction. ${ }^{18}$ As observed in our second case, other patients treated with mycophenolate mofetili9 or infliximab ${ }^{18}$ for different conditions have been found to develop primary CNS lymphomas. Finally, lymphoma can be secondary to CLIPPERS since the chronic perivascular antigenic stimulation might lead to the malignant transformation of B-cells targeting this antigen'1.

In conclusion, the specific vulnerability of an immature brain during disease onset may be the cause of the more severe phenotype seen in CLIPPERS paediatric cases. We hypothesise that early aggressive treatment prior to the development of brain atrophy and irreversible symptoms may be required for improved outcomes. Neuropathological findings suggest that Natalizumab may be the most suitable biological drug to treat CLIPPERS. However, safety issues need to be taken into consideration. ${ }^{20}$ Side effects should be discussed carefully within the clinical team and with patients, and strict clinical surveillance is always required.

\section{References}

1. Pittock SJ, Debruyne J, Krecke KN, et al. Chronic lymphocytic inflammation with pontine perivascular enhancement responsive to steroids (CLIPPERS). Brain : a journal of neurology 2010; 133(9): 2626-34.

2. Taieb G, Allou T, Labauge P. Therapeutic Approaches in CLIPPERS. Curr Treat Options Neurol 2017; 19(5): 17.

3. Simon NG, Parratt JD, Barnett $\mathrm{MH}$, et al. Expanding the clinical, radiological and neuropathological phenotype of chronic lymphocytic inflammation with pontine perivascular enhancement responsive to steroids (CLIPPERS). J Neurol Neurosurg Psychiatry 2012; 83(1): 15-22. 
4. Taieb G, Duflos C, Renard D, et al. Long-term outcomes of CLIPPERS (chronic lymphocytic inflammation with pontine perivascular enhancement responsive to steroids) in a consecutive series of 12 patients. Archives of neurology 2012; 69(7): 847-55.

5. Salam A, Sana F, Nazir R, Saeed MA, Iqbal Y, Ahmad A. Clippers (chronic lymphocytic inflammation with pontine perivascular enhancement responsive to steroids)-Case report with neuroimaging. Pakistan Journal of Neurological Sciences 2014; 9(3): 26-8.

6. Mathias S, Hickman D, Lightner D, Smith C, Baumann R. A Pediatric Case of CLIPPERS (Chronic Lymphocytic Inflammation with Pontine Perivascular Enhancement Responsive To Steroids). Neurology 2015; 84(14 Supplement P6.265).

7. Veerapandiyan A, Chaudhari A, Deo P, Ming X. Chronic lymphocytic inflammation with pontine perivascular enhancement responsive to steroids (CLIPPERS): A pediatric case report with six year follow-up. Mult Scler Relat Disord 2017; 17: 95-8.

8. Tobin WO, Guo Y, Krecke KN, et al. Diagnostic criteria for chronic lymphocytic inflammation with pontine perivascular enhancement responsive to steroids (CLIPPERS). Brain : a journal of neurology 2017; 140(9): 2415-25.

9. Gabilondo I, Saiz A, Graus F, Villoslada P. Response to immunotherapy in CLIPPERS syndrome. J Neurol 2011; 258(11): 2090-2.

10. Taieb G, Wacongne A, Renard D, Figarella-Branger D, Castelnovo G, Labauge P. A new case of chronic lymphocytic inflammation with pontine perivascular enhancement responsive to steroids with initial normal magnetic resonance imaging. Brain : a journal of neurology 2011; 134(Pt 8): e182;.

11. Blaabjerg $M$, Ruprecht $\mathrm{K}$, Sinnecker $\mathrm{T}$, et al. Widespread inflammation in CLIPPERS syndrome indicated by autopsy and ultra-high-field 7T MRI. Neurol Neuroimmunol Neuroinflamm 2016; 3(3): e226.

12. Jones JL, Dean AF, Antoun N, Scoffings DJ, Burnet NG, Coles AJ. 'Radiologically compatible CLIPPERS' may conceal a number of pathologies. Brain : a journal of neurology 2011; 134(Pt 8): e187.

13. Pfeifenbring S, Bunyan RF, Metz I, et al. Extensive acute axonal damage in pediatric multiple sclerosis lesions. Ann Neurol 2015; 77(4): 655-67.

14. Chitnis T. Disease-modifying therapy of pediatric multiple sclerosis. Neurotherapeutics 2013; 10(1): 89-96.

15. Taieb $\mathrm{G}$, Uro-Coste $\mathrm{E}$, Clanet $\mathrm{M}$, et al. A central nervous system B-cell lymphoma arising two years after initial diagnosis of CLIPPERS. J Neurol Sci 2014; 344(1-2): 224-6.

16. De Graaff HJ, Wattjes MP, Rozemuller-Kwakkel AJ, Petzold A, Killestein J. Fatal B-cell lymphoma following chronic lymphocytic inflammation with pontine perivascular enhancement responsive to steroids. JAMA Neurol 2013; 70(7): 915-8.

17. Taieb G, Renard D, Labauge P. Should CLIPPERS be considered a Prelymphoma state or a new Inflammatory disease? JAMA Neurol 2013; 70(9): 1200-1.

18. Alobaid A, Torlakovic E, Kongkham P. Primary Central Nervous System Immunomodulatory Therapy-Induced Lymphoproliferative Disorder in a Patient with Ulcerative Colitis: A Case Report and Review of the Literature. World Neurosurg 2015; 84(6): 2074 e15-9.

19. Balci MA, Pamuk GE, Unlu E, Usta U, Pamuk ON. Development of primary central nervous system lymphoma in a systemic lupus erythematosus patient after treatment with mycophenolate mofetil and review of the literature. Lupus 2017; 26(11): 1224-7.

20. Clerico $M$, Artusi CA, Di Liberto A, et al. Long-term safety evaluation of natalizumab for the treatment of multiple sclerosis. Expert Opin Drug Saf 2017; 16(8): 963-72. 


\section{Figure 1 capture:}

Brain MRI: A and B: Case 1 aged 3 years: axial T2 (A) and coronal T1 (B) weighted contrasted images showing confluent abnormal signal in the pons and adjacent cerebellar white matter with a distinctive punctate pattern of enhancement. $\mathbf{C}$ and $\mathbf{D}$ : Case 1 aged 15 years: axial T2 (C) and T1 (D) weighted contrasted images showing atrophy and gliotic scarring of the previously affected dorsal pons and persisting lesions with the punctate pattern of enhancement in the adjacent cerebellar white matter. $\mathbf{E}$ and F: Case 2: axial T2 (E) and coronal T1 (F) weighted contrasted images showing punctate enhancing foci in the cerebellum, midbrain, basal ganglia and hippocampus. $\mathbf{G}$ and $\mathbf{H}$ : Case 3: axial FLAIR contrasted images showing multiple hyperintensity regions within the left middle cerebellar peduncle and dorsal pons.

Brain biopsies: I: Case 1 biopsies showed white matter with a dense but patchy chronic inflammatory cell infiltrate composed of lymphocytes and macrophages (I-1). The inflammation was present around vessels but extending in the white matter parenchyma (I-1). There was patchy loss of myelin (I-2) and axons (I-3). The infiltrate contained a mixture of T cells, $B$ cells and macrophages with a prominent $T$ cell component (1.4).

$\mathrm{J}$ : Case 2:

H\&E - hematoxylin and eosin, LFB - luxol fast blue, NF - neurofilament. 